\title{
Part 2: Aging
}

\author{
Reducing Health Disparities in Older People \\ through Assistive Technology \\ Jeffrey W. Jutai* \\ Faculty of Health Sciences and LIFE Research Insti- \\ tute, University of Ottawa, Ottawa, Canada, KIN 6N5
}

Background: Health disparities are preventable differences in the opportunities to achieve optimal health that are experienced by socially disadvantaged populations. Older people are at a greater risk of experiencing health disparities than younger people. Technology has great potential for reducing disparities, particularly as we improve our understanding of the role it plays in social processes of aging. The next generation of assistive technologies should be informed by research on how best to ensure that they can be easily and effectively integrated with educational, health and social services, and that their benefits can be distributed equitably across all populations of persons who are ageing. Method: This author conducted a scoping review of the literature on the relationship between assistive technology and social determinants of healthy ageing. A search was conducted for journal publications of original research studies and reviews in the English language. Search engines included PubMed (Medline), CINAHL (Ebsco), PsycINFO and Web of Science, using the following parameters: aging population (including elderly and seniors); assistive technology (including communication technology and health technology); health disparities (including health inequities, social isolation, and social determinants of health).

Key results: Two social constructs are central to social relationships and physical health in ageing: social support and social integration. Social support refers to a social network's provision of psychological and material resources intended to benefit an individual's ability to cope with stress. Social integration is defined as participation in a broad range of social relationships. It is a multidimensional construct thought to include a behavioral component and a cognitive component. Assistive technology (AT) is capable of influencing both of these components. Precisely how AT influences social support and social integration, and thereby affects healthy ageing, is unknown. Assistive technology, such as information and communication technologies (ICTs) are promising technologies for improving for social support and social integration because they emphasize intervention in natural social networks. They are a means of making changes in social relationships that one can rely on months and years beyond the period when they are first introduced to an older person. ICTs can be used also to increase the availability of social support within existing social networks by helping to improve individual social skills or by building stronger ties to existing network members. They increase social integration by creating and nurturing close and peripheral ties between an older person and their community.

Conclusion: Assistive technology (AT) has the potential to intervene successfully in all critical areas of influence on social connectedness provided that three essential spheres of activity are optimally interacting: (1) Technology - the technology can demonstrably produce the effects on social support and social integration described above; (2) Services - educational, health, and social services are available to help an older person exploit these functional benefits; (3) Policy - subpopulations of older persons (e.g. cultural or linguistic minorities, ID and DD populations who are ageing) are not excluded from the provision of AT supports and services are treated equitably.

*Corresponding author: jjutai@uottawa.ca

\author{
Assistive Social Care Technologies for Older Peo- \\ ple: Let's Talk More About This \\ Alison Orrell ${ }^{\mathrm{a}, *}$ and Fiona Verity ${ }^{\mathrm{b}}$ \\ ${ }^{a}$ School of Health Care Sciences, Room 317 Fron \\ Heulog, Bangor University, Bangor, Gwynedd, LL57 \\ $2 D G$, Wales, $U K$ \\ ${ }^{\mathrm{b}}$ College of Human and Health Sciences, Room 135 \\ Haldane Building, Swansea University, Swansea, SA2 \\ 8PP, Wales, UK
}


Background: Health and social care are facing unprecedented challenges due to the changing patterns of disease, the demanding expectations of service users, financial restrictions and an ever increasing ageing population. The effect of these challenges are observed in the increases in demand for, and use of, health and social care, the cost of caring and the need for more qualified carers. Assistive technologies, in particular digital technologies, are being heralded as part of the solution to provide sustainable social care services. Human relationships are paramount in social care across the life course and are important for the care workforce as giving care often has value for the care giver. Protecting autonomy and upholding the right to human relationships is integral to treating older social care users with dignity. If assistive technologies are to be of benefit to future generations of people in need of assistance we posit that a broader community discussion about the value, worth and place of assistive technologies in social care is required to help inform and to realize policy ambitions and to meet the social care needs of older adults. The aim of this research was therefore to identify key questions for future dialogue. Method: A systematic literature search with a narrative synthesis was undertaken to identify potential research questions for debate. Using free text terms, synonyms and subject headings relating to assistive technology and social care, a systematic search of articles published in English between January 2000 and December 2018 were sourced from Medline (EBSCO), CINAHL (EBSCO), PsychINFO (ProQuest), Social Science Premium Collection (ProQuest), British Library Social Welfare Portal and the EThOS databases. Policy documents and discussion pieces on digital technology use in social care were also reviewed as they demonstrate emergent thinking around societal, ethical and moral issues. Record titles and abstracts were assessed by research team members. After removing duplicates, 2473 records were identified for review. Sixty-seven full-text copies of items thought to meet the review inclusion criteria were obtained and assessed against these criteria. Forty-two items fulfilled the inclusion criteria. Eligibility criteria were: items had to be written in English and had to describe and/or report on the use of assistive technology in a social care setting for older people. Disagreements were resolved by consensus within the research team.

Key results: Three distinct questions emerged from the data for broader discussion: (1) What are the implications of placing elders in a position where they are 'cared for' by technology devices? (2) If assistive care technologies fail to provide assistance as good as people, is it right that limited resources are invested in devices instead of the social care workforce? (3) Whose interests are being serviced: the people who are caring, the people who are being cared for or the market?

Conclusion: To ensure that the promises and benefits of assistive technologies in social care are realized a clearer understanding of the importance of relational care and people's interdependence is required by engaging a wider audience in the discussion.

Keywords: Assistive technology, Social care, Older people, Human relationships.

*Corresponding author. E-mail: a.orrell@bangor.ac.uk

\section{Experiences with the Use of Welfare Technologies for Elderly Persons}

Lisbet Grut $^{\mathrm{a}, *}$, Mette Røhne ${ }^{\mathrm{a}}$, Dag Ausen ${ }^{\mathrm{a}, \mathrm{b}}$ and Tone $\emptyset_{\text {derud }}{ }^{\mathrm{a}}$

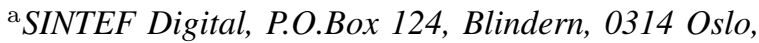
Norway

${ }^{\mathrm{b}}$ Imatis AS, Porselensvegen 14, 3920 Porsgrunn, Norway

Background: Western societies are facing challenges in health care due to the increasing number of elderly persons, persons with dementia and persons with chronic diseases. The use of technology is expected to support active aging and independent living and to reduce the need for specialised care at nursing homes. The term 'welfare technology' refer to technologies that may support persons safety, freedom and active aging, while maintaining independence and autonomy at home and comprises terms such as assistive technology, care technologies, telecare and technologies for health monitoring and medication support. The objectives of the research studies are to document how elderly persons, persons with dementia and persons with chronic diseases, and their caregivers experience the use of safety alarms, localisation technologies and medication dispensers. The article describes and discusses how the use of these technologies contributes to physical activity, safety, freedom and independent living.

Method: Action research and a participatory innovative design were chosen in order to understand underlying causes and enabling future improvement and development. The inclusion criteria were elderly persons living at home aiming for independent and active living, persons experiencing orientational challenges or persons receiving medical assistance from home- 
based services. Professional caregivers assessed each individual users' needs before inclusion and selecting the appropriate welfare technology. Informed consent was obtained from the participants or by proxy from their relatives or professional caregivers. The study included 71 participants using Mobile Safety Alarms, 208 participants using Localisation Technology (GPS) and 49 participants using Medication Dispensers. It was a four-year longitudinal study.

Key results: For users of Mobile Safety alarms, most users $(97 \%)$ reported that carrying the alarm and knowing that they might activate the alarm-button and call for help, if necessary, made them feel safe and they could continue their outdoor activities. About half of the users $(52 \%)$ reported that the alarm gave them more freedom. Safety and the users' freedom to walk outdoor alone were also the most frequently reported impacts of using the GPS (90\%), and this increased the users' autonomy and physical activity. Most relatives and professional caregivers reported that they felt safe and less anxious, because the GPS enabled them to locate and find the user, if necessary, and possibly avoid further escalation and emergency situations. $89 \%$ of the users reported that the medicine dispenser increased their sense of mastery and independence enabling self-management of medications. More than one-third (39\%) of the users of dispenser, reported increased freedom and mobility. The dispensers significantly reduced the number of home visits by professional caregivers to half of the users.

Conclusion: The studies demonstrate that welfare technologies contribute to maintaining physical activity, safety, freedom and independent living for elderly people, persons with dementia and persons with chronic diseases. These technologies facilitated the user's ability to continue living independent at home, and welfare technologies may have the potential to delay the need for transfer to nursing homes. Careful assessment of each users and close collaboration among users, relatives and professional caregivers were reported to be a prerequisite for successful implementation.

Keywords: AT for elderly persons, AT for persons with dementia, assistive technology, welfare technology, mobile safety alarm, localisation technology, GPS, active aging.

*Corresponding author. E-mail: lisbet.grut@sintef.no
Assistive Products Use Among Oldest-Old People in Japan: Differences in Personal Attributes and Living Situation

Misato Nihei ${ }^{\mathrm{a}, \mathrm{b} *}$, Ikuko Sugawara ${ }^{\mathrm{b}}$, Nozomi Ehara ${ }^{\mathrm{a}}$, Yasuyuki Gondo ${ }^{\mathrm{c}}$, Yukie Masui ${ }^{\mathrm{d}}$, Hiroki Inagaki ${ }^{\mathrm{d}}$, Takenobu Inoue ${ }^{\mathrm{e}}$, Malcolm MacLachlan ${ }^{\mathrm{f}}$ and Eilish McAuliffe ${ }^{\mathrm{g}}$

${ }^{a}$ Department of Human and Engineered Environmental Studies, The University of Tokyo, 5-1-5 Kashiwanoha, Kashiwa, Chiba, Japan

${ }^{\mathrm{b}}$ Institute of Gerontology, The University of Tokyo, 73-1 Hongo, Bunkyo Ward, Tokyo, Japan

${ }^{\mathrm{c}}$ Graduate School of Human Sciences, Osaka University, 1-2 Yamadaoka, Suita, Osaka, Japan

${ }^{\mathrm{d}}$ Tokyo Metropolitan Institute of Gerontology, 35-2 Sakae, Itabashi Ward, Tokyo, Japan

${ }^{\mathrm{e}}$ Research Institute of National Rehabilitation Center for the Persons with Disabilities, 4-1 Namiki, Tokorozawa, Saitama, Japan

${ }^{\mathrm{f}}$ Maynooth University, Maynooth, Co Kildare, Ireland

g University College Dublin, Belfield, Dublin 4, Ireland

Background: Improvements in healthcare and lifestyles have allowed more people to live longer than ever. Japan, for example, has approximately 67,000 centenarians - the highest ratio per population in the world. Increasing age, however, is associated with increase in frailty, cognitive decline, chronic illness, and other impairments, and the use of assistive products (AP) becomes essential to live comfortably and autonomously in one's home. With the number of older people worldwide forecast to increase substantially, the global demand for AP is sure to increase. The use of AP can reduce the effects of several types of impairment, allowing older people to live more comfortably at home and remain active and engaged members of society. The very old population of Japan offers a unique source of information about the use of AP later in life. In this study, we will conduct a survey of noninstitutionalized individuals, 90 years old and above, about their experiences with AP. The aim is to provide important insights to service providers in Japan, and globally, about strategies for the optimal provision of AP to older populations.

Method: Self-written or amanuensis questionnaires were posted to 4,530 people $(90+$ years) from the Kashiwa City resident basic ledger. We analyzed 1,602 valid responses $(35.4 \%)$. The mean age was $92.8 \pm$ 2.8 years old (484 males and 1,045 females); 45 persons were centenarians. The survey items are as follows: (1) age, gender, person living with, etc.; (2) liv- 
ing functions (level of nursing care needed, IADL etc.); (3) services in use (support services, AP, etc.); and (4) inconvenience in daily life (free description). This research was conducted with the approval of the WHO and the ethics review committee of each affiliated institution.

Key results: Living situation was as follows: living alone (17\%), living with spouse (11\%), living with other family members etc. $(50 \%)$, and living in facilities $(22 \%) ; 67 \%$ of respondents received some national care support services, and 1,574 people $(98.3 \%)$ are using some kind of AP. The utilization rates of dentures (76.2\%), eyeglasses $(64.2 \%)$, handrails $(41.9 \%)$, and cane $(41.0 \%)$ are high rate. There were also users of multiple AP (10+ types). Differences were found depending on AP, such as a wheelchair, which increases the utilization rate due to aging, and other AP, which decrease (hearing aid and walker). There was also a gender difference, and the utilization rate of walking AP was significantly higher for women. In addition, the usage rate of information AP for personal computers and smartphones was significantly higher for males. After further analysis to determine the reasons for these gender differences, we found that oldest-old females tended to have lower mobility than their male counterparts resulting in the higher need for walking AP.

Conclusion: The diversity of living conditions of elderly people including AP, nursing care services, and expert support was revealed. Additionally, the utilization of AP has revealed the fact that various items such as gender differences, degree of need for care, IADL, living together, etc., are involved.

Acknowledgement: This research was supported by the World Health Organization Centre for Health Development (WHO Kobe Centre - WKC: K18005).

Keywords: Assistive Product, Oldest-Old, Questionnaire Survey.

*Corresponding author. E-mail: mnihei@edu.k.utokyo.ac.jp

\footnotetext{
"Preferred" Light Color in Home Lighting Interventions for People with Age-related Macular Degeneration (ARDM)

Claude Vincent ${ }^{\mathrm{a}, \mathrm{c}, *}$, Nathalie Cimon ${ }^{\mathrm{b}}$, Paule Verville ${ }^{\mathrm{b}}$, Frédéric Dumont ${ }^{\mathrm{c}}$ and Julie Bourassa ${ }^{\mathrm{c}}$

${ }^{a}$ Department of rehabilitation, Université Laval, 1050 av. de la Médecine, Quebec city, Quebec, G1V OA6, Canada

${ }^{\mathrm{b}}$ Low vision program, Centre intégré universitaire de
}

santé et de services sociaux de la Capitale-Nationalesite de l'Institut de réadaptation en déficience physique de Québec, 525 Boul. Hamel, Quebec city, Quebec, G1M 2S8, Canada

${ }^{\mathrm{c}}$ Center of interdisciplinary research in rehabilitation and social integration, 525 Boul. Hamel, Quebec City, Quebec, G1M 2S8, Canada

Background: The level of brightness in the environment (lux) has a significant impact on the visual potential for people with visual impairments, but little is known about the impact of light colors (Kelvin). Only one descriptive study investigated the effect of light colors on reading performance $(n=107)$. It revealed better near-vision, smaller readable character size, and increased reading speed when using a magnifying glass with the preferred color temperature $(2700 \mathrm{~K}-4500 \mathrm{~K}-6500 \mathrm{~K})$. Also, no publication was found to study the effect of ambient light colors on visual comfort. Our research question focused on the evaluation of the preferred choice of ambient lighting color tested with a near-vision assessment in clinic and by using standardized tools to evaluate the impacts at home for people with age-related macular degeneration (ARMD).

Method: An explanatory quasi-experimental design was proposed with 4 evaluation times and 3 interventions. A sample of 10 ARMD patients with visual acuity between $6 / 21$ and $6 / 48$ was recruited within the visual impairment program. The intervention lasted between 8 to 10 weeks for each patient and involved two visual impairment rehabilitation specialists. Evaluations were done with the Minnesota Low-Vision Reading Test (MN Read), the International Reading Speed Texts (IReST) the visual comfort scale (0-10), a digital Lux Meter-LX1330B and the Home environment lighting assessment (HELA). During clinical interventions the following instruments were used : the LuxIQ $(4500 \mathrm{~K}-5000 \mathrm{~K}-6500 \mathrm{~K})$, magnifying glasses (diopters-10-12-16-20-24-28-3940 ), and standing models (Eschenbach ${ }^{\circledR}$ _Optelec ${ }^{\circledR}$. Schweizer $\left.{ }^{\circledR}\right)$. Home lighting interventions consisted of: addition/modification of light bulbs (fluocompactDEL-incandescent), rearrangement of lamps and glare reduction.

Key results: Six women and one man of $83 \pm 11$ years old with ARDM completed the experiment. In all cases, the use of the LuxIQ and illuminating magnifying glasses showed positive impacts in near vision to increase reading speed (49 to 58 words/min.) and visual comfort $(5 / 10$ to $8 / 10)$. It also shows positive 
impacts in ambient vision after lightning interventions with preferred color in kitchen $(n=3)$, living room $(n=3)$ and office $(n=1)$. The level of ambient lighting at home was highly increased in all cases (249 to 1295 lux). HELA satisfaction also increased (3/10 to 8/10). In all cases, the preferred light color chosen in near vision was the same as the one used for ambient lighting. The cold color $(5000-6500 \mathrm{~K})$ was chosen 5 times and once for the warm color (2700-3500 K) and the white color (3500-4000 K).

Conclusion: Our preliminary results should have an impact on visual impaired rehabilitation. So far, we have demonstrated that by relying on the "visual" aspect of color (cold, white and warm) and not just the selected Kelvin, the color of light is transferable from the near vision to the ambient lighting vision in a chosen room. The color of the "cold" light temperature seems to be the preferred color of light in rooms where activities require precision. It would be interesting to test if the chosen temperature color (cold $\pm 5000 \mathrm{~K}$ ) remained the same in a room designed for relaxing or resting activities. A multi-centric study could be a solution to realise more meaningful results at statistical level.

Keywords: Low vision, Light color, Home intervention (kitchen), Reading

*Corresponding author. E-mail: claude.vincent@rea. ulaval.ca

\section{Stay@home with Dementia: From Needs Assess- ment to Assistive Technology \\ Jorina Reekmans ${ }^{\mathrm{a}, *}$, Ryanne Lemmens ${ }^{\mathrm{a}, *}$, Steffi Rijs ${ }^{\mathrm{b}}$ and Annemie Spooren ${ }^{\mathrm{a}}$ \\ ${ }^{a}$ PXL University of Applied Sciences and Arts, Guf- fenslaan 39, 3500 Hasselt, Belgium \\ ${ }^{\mathrm{b}}$ VIVES University of Applied Sciences, Xaverianen- straat 10, 8200 Brugge, Belgium}

Background: The number of patients with dementia living at home (about 70\%) is still increasing. Assisted technology (AT) can support patients with dementia and their caregivers in daily life, to improve independence and safety. Despite the supply of AT, the use of AT in daily practice is only limited. One of the reasons is the fact that most AT is not adapted to the needs of persons with dementia. This study aims to investigate the specific needs of persons with dementia and their (in)formal caregivers in relation with AT and aging in place and to translate these needs into functional requirements.
Method used: To investigate the most important problems patients with dementia experience, patients $(n=$ 18 ), informal caregivers ( $n=72$ ) and healthcare professionals $(n=93)$ filled in the FINAH and Amsterdamse IADL questionnaire. Qualitative research using focus groups and semi-structured interviews with 6 informal caregivers and 20 healthcare professionals, provided detailed information about the problem activities, their barriers and requirements for AT.

Key results: Based on the questionnaires and the focus groups, the 10 most important problem activities included: using appliances (e.g. household appliances, TV remote, telephone), disorientation (in time/structure of the day and regarding location), household activities (e.g. preparing meals, doing groceries, doing the laundry), administration (e.g. making payments, fill in forms, keep an appointment), personal care (e.g. washing oneself, toileting), taking medicines, emotions and behaviour (e.g. being depressed, disinhibition, fear, aggression), social contacts/participation and taking care of their own health. Barriers within these problem activities were in the following categories: insight/perception, structure, habits, behaviour/emotions, disorientation, complexity, recognition, preservation of independence, forgetfulness, learning new things, safety, not performing, social participation, co-morbidity, professional care and distraction. These barriers were translated into functional requirements of assistive technology.

Example:

Problem activity: using appliances (TV remote)

One of the barriers: complexity, i.e. too many buttons, too many different remote controls, too many options/functionalities, difficult operation.

Functional requirements: use limited number of buttons, reduce the number of steps to be taken to a minimum to operate the remote, make the remote adaptable to individual needs, make it impossible to disorganize the apparatus.

Additionally, some general requirements for assisted technology for persons with dementia were formulated in the following categories: Usage (e.g. userfriendliness, working autonomously, durability/ sustainability, easily accessible, reliability), design (e.g. recognizable, (un)conspicuous, neutral, visual, integrated), flexibility (e.g. tailor-made, adaptable to stage of dementia, suitable for home situation), aimed effect (e.g. safety, independence, reassurance, burden informal caregivers, social participation, communication), privacy and security (e.g. data security, privacy), juristically, financial (e.g. price, leasing possibilities) 
and implementation (e.g. practicing with AT, timing, role (informal) caregiver, requirements home).

Conclusion: This exploratory study gives an overview of the most important problem activities for patients with dementia, the barriers within these activities and the requirements for assistive technology. The results were reported to companies specialized in assistive technology to support user-centred-design. In the second phase of this study, companies and healthcare professionals are working together in cocreation to develop/adapt products to create a user-centred solution for a problem activity identified in this study.

Keywords: Assistive technology, dementia, needs assessment

*Corresponding author. E-mail: Ryanne.lemmens@ pxl.be

\section{CRDL - Interactive Technology Eliciting Engage- ment in Elderly People with Dementia}

Renée van den Heuvel* ${ }^{*}$ Monique Lexis and Ramon Daniëls

${ }^{a}$ Zuyd University of Applied Sciences, Research centre for assistive technology in care, Nieuw Eyckholt 300, 6419 DJ Heerlen, The Netherlands

Background: People with an advanced stage of dementia encounter problems with communication and verbal interaction, leading to a decrease in participation and social isolation. CRDL (Cradle) is an interactive instrument, specifically developed for people with dementia to stimulate communication and social interaction. Based on different types of touch, CRDL produces sounds. Research on the effects of CRDL to stimulate engagement is lacking. The research questions of this study were as follows:

1. Which interventions using CRDL may contribute to the stimulation of engagement for people with dementia?

2. What are the effects of these interventions in promoting engagement in people with dementia?

Method: The study consisted of two phases using a mixed-methods design. First, two interventions with CRDL aiming at the promotion of engagement were developed based on results of semi-structured individual interviews with 9 professionals and a brainstorm session with 6 professionals from elderly care organizations in the Netherlands. Second, the interventions were tested in daily practice in two nursing homes with 30 clients following an ABB-design; phase A includes one intervention session without CRDL; phase
B (B1 and B2) includes interventions with CRDL. The primary outcome 'engagement' was measured with the Positive Response Schedule (PRS), an instrument developed to score engagement based on videoobservations. Moreover, interviews with participating professionals were conducted to evaluate experiences of using CRDL. PRS data were analyzed using the Wilcoxon signed ranked test by comparing scores on the individual items between sessions A-B1 and A-B2. Key results: The two interventions developed were 1) 'influencing behaviour' and 2) 'promotion of social interaction'. The procedure on how to perform the interventions was described in a manual. In phase 2 of the study 30 clients with severe dementia (22 female, 8 male) and six care professionals of two elderly care organizations participated. The professionals could choose intervention 1 or 2 . For 10 clients intervention 1 was selected and for 20 clients intervention 2. In the A-phase interventions without CRDL were used. For the intervention 'influencing behaviour' positive effects were found compared to interventions without CRDL on four items of the PRS, i.e. deliberate body movement (B1-A $p=0.051$ and B2-A $p=$ $0.025)$, deliberate head movement (B1-A $p=0.017$ ), focus on environment (B1-A $p=0.011$ and $\mathrm{B} 2-\mathrm{A} p=$ $0.091)$ and engagement (B1-A $p=0.059$ and B2-A $p=0.097)$. No differences were found on the PRS items for the intervention 'promotion of social interaction'. Professionals all reported positive experiences of using CRDL and expressed their willingness to proceed working with CRDL.

Conclusion: The study resulted in two meaningful interventions for application of CRDL in dementia care practice and gave positive indications for effects of CRDL to stimulate engagement. Future research on the effects of these and other CRDL interventions among a larger population and in other target populations, such as people with intellectual disabilities is recommended.

Keywords: CRDL, technology, dementia, engagement *Corresponding author. E-mail: renee.vandenheuvel@ zuyd.nl

Assistive Technologies for Older Persons with Intellectual Disabilities. A Preliminary Systematic Review for Future Research Implementation

Mabel Giraldo ${ }^{\mathrm{a}, *}$, Serenella Besio ${ }^{\mathrm{a}}$ and Patrizia Marti ${ }^{\mathrm{b}}$ ${ }^{a}$ Department of Human and Social Sciences, University of Bergamo, Piazzale S. Agostino 2, 24129 Bergamo, Italy 
${ }^{\mathrm{b}}$ Department of Social, Political and Cognitive Science, University of Siena, Via Roma 56, 53100 Siena, Italy

Background: The number of older persons, including those with disabilities, has increased substantially in most countries and it will accelerate in the next decades. Among this population, experts have established a conceptual and factual distinction between two different phenomena: disability with aging (elderly incurring into some kind of impairments) and aging with disability (elderly who incurred into impairments during the developmental age). In both cases, assistive technologies (AT) can play an important role in supporting and stimulating elderly with disabilities in order to improve their quality of life and to support them in cognitive and social skills maintenance. In particular, the present paper aims at mapping the state of the art in ATs developed in the last 5 years for older persons with intellectual disabilities (ID).

Method: Adopting PRISMA checklist, a systematic review was conducted in IEEE Digital Library, ACM Digital Library and Scopus databases using the following keywords: a. assistive technology OR assistive devices OR domotics; b. accessib*; c. disabil* older people OR disab* elderly; $d$. intellectual disability OR mental retardation; period: 2014 to 2019. Inclusion criteria were scientific papers, proceedings and book chapters addressing the topic of interest. Studies that did not include older adults/elderly with ID and researches that did not focus on the use of ATs and related devices (indoor/outdoor, embodied, etc.) for these target-users were excluded.

Key results: After an initial selection of 520 articles, 61 were subjected to in-depth analysis leading to the final selection of 45 papers. The selected contributions reflect a heterogeneous variety of ATs (prototypes, on-purpose/low cost/commercial devices, etc.) that can support the older persons with cognitive impairments: advanced sensors/networks (n.10); cognitive assistants (n.3); robots (n.3); wireless communication systems (n.14); wearable technologies (n.6). Moreover, some papers (n.5) collect or combine different kind of devices while a few (n.4) face the economic, ethical and legal issues connected to the use of ATs with older/disabled persons. In the majority of the studies, these ATs were developed for older persons who acquired some kind of cognitive impairments (e.g. dementia) in older age (n.18) or, according to their designers, could be used both for age-related cognitive disorders and for older persons with life-long ID (n.20). Only a few studies (n.7) focused on the use of ATs for persons aging with ID.

Conclusion: The paper reports on the increasing number of ATs designed to assist elderly with cognitive disabilities to meet their needs. Simultaneously, it highlights that only few researches focus on AT devices for persons aging with ID. This confirms the lack of theoretical/concrete integration between aging and disability studies. These aspects, together with premature aging (generally associated with ID), result in limited understanding of how the experience of living with a long-term disability influences the experience of aging. Consequently, more attention to, and the development of ATs specifically designed for the aging population with ID is needed. New challenges for future researches will be addressed and further possible exploitation/application for supporting "successful aging with disabilities" will be drawn.

Keywords: Intellectual disabilities, Older person, Aging with disability, Assistive Technologies.

*Corresponding author. E-mail: mabel.giraldo@unibg. it

\section{Virtual Garden for People with Dementia}

Anne-mie Sponselee ${ }^{\mathrm{a}, *}$ and Astrid Dooremalen ${ }^{\mathrm{a}}$

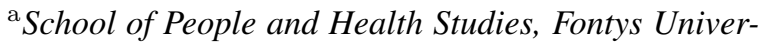
sity of Applied Sciences, Postbus 347, 5600 AH, Eindhoven, The Netherlands

Background: SENSE-GARDENs will strengthen the awareness of older people with dementia by providing stimuli to the different senses, such as sight, touch, hearing, balance and smell. In this study, several prototypes of the AAL-project were tested. For the RealityWall - a large screen showing film and photos, with sounds and music, and matching vibrations - the content must be tuned to a person's cultural background. In this study the desires and needs of Dutch older people with dementia in relation to the Reality-Wall were researched, with the goal to have content for the RealityWall when implemented in the Netherlands. The Liferoad/Cycling-Home consists of a stationary bicycle in front of a screen on which the environment of the cycle route is presented. In this study, various technological set-ups were tested with the goal to increase the sense of immersion to improve the well-being of people with dementia.

Method: In co-creation with nine people with dementia (phase CDR-2 and CDR-3) and their family, moodboards were created illustrating what kind 
of films, music, tv-shows, art, etcetera would fit peoples' interests and bring up good memories. Based upon these moodboards, a 24-minute film with 21 fragments in four categories (TV; photo with sound; TV with music; photo) was created to test, using participatory observations. Five people with dementia (phase CDR-2 and CDR-3) were shown the film twice (with and without a family member), while researchers measured the number of verbal/non-verbal, social/non-social behavior for each fragment, using the Verbal and Nonverbal Interaction Scale. For the Liferoad/Cycling-Home twenty care-home residents with dementia were randomly attributed to two out of four test settings (Google Streetview (personal choice of environment); 360-degrees video of the care-home environment; Bikelabyrinth (commercially available interactive routes); ClubVirtual (commercially available virtual cycling routes). Participants were seated in front of a stationary bicycle and a television screen, and were cycling for three minutes per test setting. Two researchers made observations, using the Discomfort Scale - Dementia of Alzheimer Type (DS-DAT), using a 5-point frequency scale.

Key results: During the 24-minute film for the RealityWall, participants mainly showed non-verbal social behavior. TV fragments with music resulted in the strongest reactions $(\mathrm{F}=6.66, \mathrm{df}=3, p<0.01)$. In comparison to observations without a family member, participants showed more verbal social behavior $(t=$ -2.54 , df $=20, p<0.05)$, more non-verbal social behavior $(t=-2.22$, df $=20, p<0.05)$, and less non-verbal non-social behavior $(t=4.26$, $\mathrm{df}=20$, $p<0.00$ ), when a family member was present during the 24-minute film. For the Life-road/Cycling-Home test, the 360 degrees video resulted in the lowest average discomfort score (DS-DAT score: 7.2). The Google Streetview setting resulted in the highest discomfort when tested first (DS-DAT: 11.2), but the lowest discomfort when tested second (DS-DAT: $5.9 ; t=2.10$; df $=8 ; p<0.10)$.

Conclusion: All fragments resulted in more social than non-social behavior, some fragments stronger than others. The presence of family members increased verbal social behavior. The film seems suitable for residents with dementia of Dutch care-homes. 360Degrees videos are useful for the Life-road/CyclingHome prototype. Participants experience no extra value in interactive routes.

Keywords: Dementia, Virtual Environment, Cocreation, Stimuli.

*Corresponding author. E-mail: a.sponselee@fontys.nl
Dementia Dogs and their Impact on Communitydwelling Persons with Mild to Moderate Dementia and their Family Caregivers

Claude Vincent ${ }^{\mathrm{a}, \mathrm{b} *}$, Frédéric Dumont ${ }^{\mathrm{b}}$, Bertrand Achou $^{\mathrm{b}}$, Cary Brown ${ }^{\mathrm{c}}$, Suzette Bremault-Phillips ${ }^{\mathrm{c}}$, Bernie Travis $^{\mathrm{d}}$, Annette Rivard ${ }^{\mathrm{c}}$ and Julie Bourassa ${ }^{\mathrm{b}}$

${ }^{a}$ Department of Rehabilitation, Université Laval, 1050 av. de la Médecine, Quebec city, Quebec, GIV 0A6, Canada

${ }^{\mathrm{b}}$ Center of interdisciplinary research in rehabilitation and social integration, 525 Boul. Hamel, Quebec City, Quebec, G1M 2S8, Canada

${ }^{\mathrm{c}}$ Department of Occupational Therapy, University of Alberta, 116 St. and 85 Ave., Edmonton, AB, T6G 2R3, Canada

${ }^{\mathrm{d}}$ Early Onset Dementia Alberta Foundation, Edmonton, Alberta, Canada

Background: Individuals with mild to moderate dementia, and their caregivers, may benefit from the support of a dementia dog. It falls into the category of cognitive aids (assistive technology for disabilities). It's a dog trained to provide cues (and sometimes reminders) and companionship, improve activity-levels and wayfinding, and enhance well-being and connection. There are very few schools that have a formal training program to train dementia dogs; we have found one dog schools in USA, one in Scotland and one in Australia. Since there is no scientific evidence addressing the efficacy of dementia dogs, our study proposes the following research objectives: 1-to examine the impacts of canine assistance on both the person with dementia and his/her caregiver (e.g., on engagement, socialization, well-being, activity levels, wandering, sense of meaning and purpose, quality of life, stress and health); 2-to conduct a cost-effectiveness analysis associated with having a canine assistance in the home of the person with dementia (e.g., costs related to dog acquisition and community training, postplacement, costs savings; benefits measured in terms of quality of life for caregivers and those with dementia); and 3-to examine the acceptance of canine assistance in public places in the prior 3 months from the caregivers' point of view (e.g. approachability situations and socialisation experiences).

Method: A comparative analysis of 3 'cases' is underway. Each case will be comprised of 20-40 dyads of a caregiver, person with mild to moderate dementia, and either (1) a companion dog, (2) a certified support dog, or (3) no dog. Data collection will include phone/Skype interviews with caregivers, a cost- 
effectiveness analysis and observation at dog training schools. Phone questionnaires used are the Concern for the Care recipient's Well-Being (CCWB) and the Impact on the Caregiver's Social Life (ICSL) (two subscales of the Caregiver's Burden Scale). Online questionnaires used are the Adult social care outcomes toolkit (ASCOT), the Social care-related quality of life (SCRQoL), the ICEpop (Investigating Choice Experiments for the Preferences of Older People) and the CAPability measure for Older people (ICECAP-O). A qualitative thematic analysis of interviews and comparisons of the quantitative data across the 3 cases will be conducted.

Key results: Preliminary results on canine assistance has a positive impact on the lives of persons with dementia and their caregivers (supporting engagement, quality of life, well-being of the dyads, and enabling the person with dementia to remain at home safely and the caregiver remain or return at work).

Conclusion: With more results about canine assistance, it may be possible to advocate for the establishment of new training programs for certificated dementia dogs across Canada. Furthermore, positive results from the cost-effectiveness analysis and acceptance of the dementia dogs in public places will inform policymakers about the feasibility of funding certified dogs and to establish the associated public policies.

Keywords: Cognitive aid, Emotional support dog, Alzheimer, Home support, Caregiver facilitator.

*Corresponding author. E-mail: claude.vincent@rea. ulaval.ca

\author{
Family Caregivers' Experience of Care and Use of \\ Assistive Technologies \\ François Routhier $^{\mathrm{a}, \mathrm{b}, *}$, Maude Beaudoin ${ }^{\mathrm{a}, \mathrm{b}}$, Oladele A \\ Atoyebi $^{\mathrm{c}}$, Claudine Auger ${ }^{\mathrm{d}}$, Louise Demers ${ }^{\mathrm{d}}$, Andrew \\ Wister $^{\mathrm{e}}$, Janet Fast ${ }^{\mathrm{f}}$, Paula Rushton ${ }^{\mathrm{d}}$, Josiane Lettre $^{\mathrm{a}}$, \\ Michèle Plante ${ }^{\mathrm{g}}$ and W. Ben Mortenson ${ }^{\mathrm{c}}$ \\ ${ }^{a}$ Centre for interdisciplinary research in rehabilitation \\ and social integration, Centre intégré de santé et de \\ services sociaux de la Capitale-Nationale, 525 Hamel \\ est, Québec, G1M 2S8, Canada \\ ${ }^{\mathrm{b}}$ Department of rehabilitation, Université Laval, \\ Québec, G1V 0A6, Canada \\ ${ }^{\mathrm{c}}$ Department of Occupational Science and Occupa- \\ tional Therapy, University of British Columbia, Van- \\ couver, V6T 2B5, Canada \\ ${ }^{\mathrm{d}}$ School of rehabilitation, Université de Montréal, \\ Montréal, H3N 1X7, Canada \\ ${ }^{\mathrm{e}}$ Department of Gerontology, Simon Fraser University,
}

Vancouver, V6B 5K3, Canada

${ }_{\mathrm{f}}^{\mathrm{f}}$ Department of Human Ecology, University of Alberta, Edmonton, T6G 2P5, Canada

${ }^{\mathrm{g}}$ Research Centre, Institut universitaire de gériatrie de Montréal, Montréal, H3W 1W4, Canada

Background: Nearly half of the Canadians will be family caregivers (individual who provides unpaid assistance to someone living with a chronic condition) at some point in their lives. Family caregivers provide $75 \%$ of the care needed for individuals with disabilities to remain in their communities. Caregiving has psychological, physical and financial consequences. Considering issues associated with caregiving, finding ways to reduce caregiver burden is critical. Research has shown that the use of assistive technologies could decrease caregiver burden. However, it is essential to know how caregivers experience care and use of assistive technologies. This knowledge would guide the development of assistive technologies that are appropriate to their situation. This study aimed to explore the experience of caregiving for older adults, whether assistive technologies are currently integrated in the care and their perceived impacts.

Method: This study draws on data from a larger multisite, mixed-methods project on Canadian family caregivers. Sites of recruitment and data collection are Vancouver (English-speaking participants) and Québec City (French-speaking participants). To be recruited, family caregivers had to speak French or English, be at least 19 years old and provide care to an older adult, or be an older adult providing care. Participants were selected during recruitment to obtain a maximum theoretical variation in terms of impairments of the person for who care was provided (i.e., physical and/or cognitive), relationship with the care recipient (e.g. spouse, child, etc.), the amount of care provision and region in Canada. Between November 2015 and October 2017, 59 family caregivers were recruited and participated in an individual semi-structured interview on their current caregiving situation and their use of assistive technologies. Participants were informed that, when asked about assistive technologies, they could talk about services (e.g. home care services), policies (e.g. care funding) and assistive devices. A thematic content analysis was conducted.

Key results: Three main themes were identified from the interviews that describe family caregivers' experiences of care. 'Responsibilities of Caregiving' described that caregivers assisted in all areas of their care recipient's life, but some factors modulated the specific 
activities for which help was provided and the type of help. An example of a modulating factor was their care recipients desire for autonomy. 'Caregivers' Challenges and Rewards' portrayed the daily challenges experienced by caregivers, such as physical strain, mental difficulties, the need for external assistance, etc. but also identified positive caregiving activities. 'Strategies to Address Responsibilities and Challenges' illustrated that caregivers use a variety of strategies to face their daily challenges, including the use of assistive technologies, but some needs are not fully addressed.

Conclusion: We developed a better understanding of the complexity of care provision, including daily challenges and needs that are not fully addressed, which may be affected via appropriate access to and use of effective assistive technologies. This study is a first step toward user-centred design research to develop innovative assistive technology solutions and areas where advocacy is needed to improve access to existing devices and services.

Keywords: assistive technology, caregiver, older adults, needs, mixed-method, interview.

${ }^{*}$ Corresponding author. E-mail: Francois.Routhier@ rea.ulaval.ca

Evaluation of an Assistive Technologies Bundle by Informal Carers of Older Adults. Results from a Pilot Trial in Austria and Italy

Ines Simbrig $^{\mathrm{a}, *}$ and Judith Kathrein ${ }^{\mathrm{b}}$

${ }^{a}$ Institute for Public Management, Eurac Research, Viale Druso 1, 39100 Bolzano, Italy

${ }^{\mathrm{b}}$ Institute for Strategic Management, Marketing and Tourism, University of Innsbruck, Universitätsstraße 15, 6020 Innsbruck, Austria

Background: There are many assistive and smart home technologies, but a residential gateway that connects them and thus creates an interoperable and adaptable technology bundle for older adults is still missing. Within the project gAALaxy, funded by the European AAL programme, such a bundle was developed and evaluated. The main research questions regarding informal carers focused on the usability of the bundle, perceived effects on well-being and quality of life variables, as well as different care aspects.

Method: The technologies were evaluated in an exploratory field study. Community-dwelling older adults and their informal carers from Austria and Italy tested a bundle, consisting of an emergency watch, a fall detector and a smart home system, for six up to 18 months.
The trial period was conducted in two phases, with the middleware of the bundle being improved before the second phase. Here, we present parts of the results, i.e. the analyses of a questionnaire filled in by 38 informal carers. It measured attitudes towards technology, the perceived impact of the bundle on the older adults and themselves, and the perceived usability and ease of use of the bundle. In pilot phase two we also asked participants about their willingness to pay for the technologies.

Key results: Statistical analyses showed that there were virtually no significant differences between phase 1 and 2 regarding the perceptions of informal carers. Surveying the impact of the bundle on different variables across both phases, we found mostly positive values though. The perceived effects on care quality, ease of use, peace of mind, comfort, safety, autonomy, and social interaction were all positive, with a total mean of 1.14 (SD: 0.64 , seven-point scale from -3 to +3 ). The mean perceived usability of the bundle was 1.63 (SD: 0.89 , seven-point scale from -3 to +3 ). The ability of the bundle to recognise emergencies quickly was rated especially positively (mean: 1.45 , SD: 0.81 , five-point scale from -2 to +2 ). Only the perceived effort of using the bundle as an informal carer was rated slightly negatively (mean: -0.62 , SD: 0.92 , five-point scale from -2 to +2 ). 10 informal carers reported some future willingness to pay a monthly fee for the whole bundle including services, with a wide range of acceptable monthly fees being stated (mean: $57.19 e$, SD: 52.57e).

Conclusion: The lack of differences between phase 1 and 2 in terms of general technology attitude and evaluation of the bundle is not surprising as the bundle changed little over time from the end-users' point of view (the improvements were of a technical nature and had no noteworthy effects). Even if there were no statistically significant increases of the perceived quality and effects of the technology bundle over time, we can still state that the overall perception by the informal carers was almost always positive in both phases. The exploratory nature of the research design did not allow sophisticated statistical analysis methods. We therefore consider the results as preparatory for bigger scale projects (e.g. i-evAALution, also funded by the AAL programme).

Keywords: Assistive Technologies, AAL, Older Adults, Informal Carers

${ }^{*}$ Corresponding author. E-mail: ines.simbrig@eurac. edu 
Evaluation of Daytime Activities at Home for Elderly Hemiplegic Patients and Development of Bed with Standing up Function to Prevent Disuse Syndrome

Katsuhiro Manabe ${ }^{\mathrm{a}}$, Tsutomu Hashizume ${ }^{\mathrm{b}}$, Taizo Shiomi $^{\mathrm{b}}$, Satoshi Miyasita ${ }^{\mathrm{b}}$, Toru Maesono ${ }^{\mathrm{c}}$ and Tsutomu Abe $\mathrm{A}^{\mathrm{d}}$

${ }^{a}$ Faculty of Medical Science Dept., Teikyo University of Science, 2-2-1 Senjusakuragi, Adachi-ku Tokyo, Japan

${ }^{\mathrm{b}}$ Faculty of Medical Science Dept., Teikyo University of Science, Tokyo, Japan

${ }^{\mathrm{c}}$ Ageo Central Medical Group, Japan

${ }^{\mathrm{d}}$ Rehabilitation Progressive Center-co, Japan

Background: The rehabilitation service of elderly hemiplegics patients at home to prevent disuse syndrome play an important role in Japan. However, the actual activity level of patients and the effectiveness of the rehabilitation are not clarified objectively. The purpose of this study is to evaluate their activities with Activity Monitoring Evaluation System (AMES) and to develop the bed with standing up function to prevent disuse syndrome. The bed can take the forms of very adjustable profile electrically, so that the patient is able to achieve a standing position through a sitting position from horizontal lying position.

Method: We examined the activities of 30 hemiplegic patients (71 \pm 3.5 years, 22 males, 8 females) with AMES while 8 hours ( 9 am to $5 \mathrm{pm}$ ) for 2 days. AMES could derive the activities such as walking, propelling wheelchair, standing, sitting, and lying position on bed. We evaluated total duration time, percentage and maximum continuous time of each activity. In order to verify the efficiency of the bed in almost bedridden another 2 hemiplegic patients ( 83 years male, 87 years female), to take standing position for 30 minutes was performed twice a day for 30 days.

Key results: 1. Mean total duration time, standard deviation were that walking: $55 \pm 37 \mathrm{~min}$, propelling wheelchair: $33 \pm 34 \mathrm{~min}$, standing: $27 \pm 21 \mathrm{~min}$, sitting: $270 \pm 100 \mathrm{~min}$, lying position: $116 \pm 119 \mathrm{~min} .2$. The mean percentage of each activity against to 8 hours were that walking: $12 \%$, propelling wheelchair: $7 \%$, standing: $6 \%$, sitting: $56 \%$, lying position: $24 \%$. 3 . Mean maximum continuous duration time and maximum time were that walking: $6 \mathrm{~min}(27 \mathrm{~min})$, propelling wheelchair: 49 seconds (1.5 min), standing: 2 min (9 min), sitting: $43 \mathrm{~min}$ ( 2 h $14 \mathrm{~min}$ ), lying position: $43 \mathrm{~min}$ ( $2 \mathrm{~h} 40 \mathrm{~min}$ ). 4 . The standing up with the bed increased serum proteins and hemoglobin that indicated the improvement of anemia and nutritional state, and improved edema in the lower extremities and ADL level. However, these results showed only slight improvement, with no statistically significant differences.

Conclusion: The percentage of walking and standing is only $18 \%$, whereas that of sitting and lying position is about $80 \%$. Nine subjects show that the percentage of walking is lower than $10 \%$. The percentage of sitting time $56 \%$ shows that the subjects have been sitting half in a daytime. The mean percentage $24 \%$ of lying position indicates that some subjects are staying in bed through the daytime, and 9 subjects have been lying for over 3 hours. The results reveal that the amount of daily activities of the hemiplegic patients in a daytime is unexpectedly low, and predict those patients may be caused a disuse syndrome. The standing up function of the bed is clinically useful, for selected patients who could show good prognosis in ADL stage. However, the primary purpose of the bed is to facilitate a change from a supine position to a sitting position and to lead from a standing position to walk. So, further study to verify the effect of the multi adjustable forms bed with AMES for improving the activity level of hemiplegic patients is required.

Keywords: Elderly hemiplegia, Daytime activities, Rehabilitation service, Standing up bed, Disuse syndrome.

*Corresponding author. E-mail: k-manabe@ntu.ac.jp

\section{Detecting Social Interaction in a Smart Environ- ment}

Andrea Masciadri ${ }^{\mathrm{a}}$, Stefano Brusadelli $^{\mathrm{a}}$, Andrea Tocchetti $^{\mathrm{a}}$, Sara Comai ${ }^{\mathrm{a}, *}$ and Fabio Salice ${ }^{\mathrm{a}}$

${ }^{a}$ Dipartimento di Elettronica, Informazione e Bioingegneria, Politecnico di Milano, Via Francesco Anzani 42, 22100 Como, Italy

Background: Over the last few decades, numerous articles and studies have shown the relationship between loneliness and its effects on people's health, especially in the case of senior citizens. Detecting loneliness requires a meticulous and continuous observation of the subject by clinicians. However, considering the growth of the population over sixty, the huge demand for health-care facilities, and the necessity to contain the costs of hospitalization, the technology is now called to support the activities of specialists. An open challenge is to measure the level of social interaction among people, and to infer a personal "loneli- 
ness" index. We propose a system to identify the interactions among people who are sharing the dining table in a smart environment.

Method: This study refers to an experimental investigation conducted in "Il paese ritrovato", an innovative health-care facility located in Monza, Italy, that assists people affected by Alzheimer Disease. The system detects the patients who are sitting around a table and their position; this information is computed integrating an environmental Bluetooth indoor localization system and an RFID module installed on the table. Moreover, the system detects the social interactions: a quad-microphone array expansion board for Raspberry Pi called ReSpeaker Mic 4-Array detects the direction from which someone is speaking. A dedicated software filters the data locally, and computes a "social index" among the people who are sitting around at the table. The communication between the main module and the devices has been implemented using MQTT, a publish/subscribe mechanism.

Key results: The experiment has been carried out in an indoor environment, by placing the system on a foursided square table with four people, one for each seat. During the testing phase, the system has been tested under three different environmental conditions: in absence of noise and voices aside from the speaker, in presence of noise and with other people speaking in the surroundings of the system. Overall, the system shows promising results (precision: $84 \%$, recall: $93 \%$, accuracy:79\%) only, in presence of loud noise, the system is still not able to identify with precision the people who participate in the interaction. We strongly believe that the computed index constitutes a valuable source of knowledge for further data analysis modules (such as the one installed in "Il paese ritrovato") to monitor patients.

Conclusion: A novel system to identify the interactions among people who are sharing the dining table in a smart environment has been presented. Thanks to a localization module based on both Bluetooth and RFID technologies, and a microphone, this system is capable to measure the level of social interaction among people. As future work, we plan to investigate the possibility to extend the system to detect social interactions in wide open spaces through the integration of a device capable of recognizing the speakers' voiceprints.

Keywords: Wellbeing assessment, Assistive Technologies, Social interaction, Smart Environments, IoT. *Corresponding author. E-mail: sara.comai@polimi.it
Continuous Ambient In-home Walking Speed Monitoring in Frail Older Adults: Results of a Feasibility Study

Phil Joddrell $^{\mathrm{a}, *}$, Stephen Potter ${ }^{\mathrm{a}}$, Luc P. de Witte ${ }^{\mathrm{a}}$ and Mark Hawley ${ }^{\mathrm{a}}$

${ }^{a}$ Centre for Assistive Technology and Connected Healthcare, School of Health and Related Research, The University of Sheffield, 217 Portobello, Sheffield S1 $4 D P, U K$

Background: Walking speed has been shown to be predictive of important clinical outcomes in older adults and has been labelled 'the sixth vital sign'. Furthermore, it has particular value as one of the most significant indicators of frailty. Despite the clinical importance of walking speed measurement (and its inclusion in guidelines regarding frailty, multi-morbidity etc.), measurement of walking speed is rarely carried out. It is not feasible to carry out clinic-based walking speed measurements with sufficient frequency to capture the rate of change of health in older people and as a result important heath deterioration is missed and health services end up reacting to crisis situations (e.g. falls). Our aim was to test whether it is feasible to measure walking speed frequently and unobtrusively in the home, using ambient technology that is easy to install and reliable (therefore usable in a health and social care setting), low cost, and acceptable to older people.

Method: A longitudinal feasibility study was conducted involving 20 older adults (aged 65 or above) living alone, identified as either frail or pre-frail by the Rockwood Clinical Frailty Scale (score between four and six). Self-selection sampling was employed within the populations of two supported-living services in Sheffield, UK. Sensors were installed in the participants' homes for a period of three months to continuously measure their walking speed. The researcher visited each participant at four time points (baseline and monthly intervals) to conduct manual walking speed measurements, administer the Rockwood Clinical Frailty Scale and a brief health interview, and collect the data from the memory cards of each device. Qualitative interviews were also conducted at the end of the study to assess participants' attitudes to the sensors and the concept of continuous in-home walking speed measurement.

Key results: Eighteen participants completed the study, with two withdrawing, one due to personal preference at month one and a second due to a change in living circumstances at month three. The number of walking speed measurements recorded by the sen- 
sors varied significantly between participants (mean 1159.39, range 4-3636); the number was determined by the placement of the device within each home and technical disruptions to the sensor operation, as well as the individual's lifestyle and degree of mobility. A near-normal distribution of speeds was evident for several participants for whom placement was good and disruption was minimal. Qualitative interviews with the participants indicated both acceptability of the device within the home environment and of the concept of measuring walking speed as an indicator of physical health.

Conclusion: This study has demonstrated the feasibility of continuous ambient in-home walking speed monitoring in older adults. Further development of the walking speed sensor is required to increase placement flexibility, improve accuracy and allow for wireless integration with online services. This will lead to a device that can be used both to support selfmanagement of frailty and monitoring of frailty by healthcare providers.

Keywords: Frailty, Walking speed, Ambient sensor technology.

*Corresponding author. E-mail: p.joddrell@sheffield. ac.uk

Effectiveness of Sensor Monitoring in a Rehabilitation Program for Older Patients After Hip Fracture: A Three-arm Stepped Wedge Randomized Trial

Margriet Pol ${ }^{\mathrm{a}, *}$, Gerben ter Riet ${ }^{\mathrm{b}}$, Margo van Hartingsveldt $^{\mathrm{a}}$, Ben Kröse ${ }^{\mathrm{c}, \mathrm{d}}$ and Bianca M. Buurman ${ }^{\mathrm{a}, \mathrm{e}}$

a ACHIEVE, Centre of Applied Research, Faculty of Health, Amsterdam University of Applied Sciences, Amsterdam, The Netherlands

${ }^{\mathrm{b} D e p a r t m e n t}$ General Practice, Amsterdam UMC, University of Amsterdam, The Netherlands

${ }^{\mathrm{c}}$ Research Group Digital Life, Amsterdam University of Applied Sciences, University of Amsterdam, Amsterdam, The Netherlands

${ }^{\mathrm{d}}$ Informatics Institute, University of Amsterdam, Amsterdam, The Netherlands

e Section of Geriatric Medicine, Department of Internal Medicine, Amsterdam UMC, University of Amsterdam, Amsterdam, The Netherlands

Background: The performance of activities of daily living (ADL) at home is important for the recovery of older persons after hip fracture. Fear of falling hinders older persons to perform ADLs. Sensor monitor- ing can be used as a coaching tool to reduce fear of falling and increase daily functioning. However, as far as we know, sensor monitoring-based programs have not yet been used in rehabilitation for older patients after hip fracture. In this randomized trial, we tested the effects of an intervention involving sensor monitoring informed occupational therapy on top of a cognitive behavioral treatment (CBT) based coaching program on patient- reported daily functioning in older patients after hip fracture.

Method used: The study was a three-armed randomized stepped wedge trial in six skilled nursing facilities, with assessments at baseline (during admission) and after one, four and six months (at home). Eligible patients were hip fracture patients 65 years and older. Patients received care as usual, CBT-based occupational therapy or CBT-based occupational therapy with sensor monitoring. The sensor monitoring system comprises 1) a wearable physical activity monitor (PAM-sensor) that is worn on the hip; 2) a network of ambient sensors comprises (Benext )passive infrared motion sensors placed in the main spaces in the patients' house and 3) a gateway, that is a raspberry $\mathrm{Pi}$ with a Z-wave shield for communication with the ambient sensors, a Bluetooth adaptor for communication with the PAM and a $4 \mathrm{G}$ dongle to make connection with the remote server. Via a web-based application users can login to the server and see the visualizations. Interventions comprised a weekly session during institutionalization, followed by four home visits and four telephone consultations over three months. The primary outcome was patient reported daily functioning at six months, measured with the Canadian Occupational Performance Measure.

Key results: A total of 240 patients (mean[SD] age, 83.8[6.9] years were enrolled. At baseline, the mean Canadian Occupational Performance Measure scores (range 1-10) were 2.92 (SE 0.20) and 3.09 (SE 0.21) for the care as usual and CBT-based occupational therapy with sensor monitoring groups, respectively. At six months, these values were 6.42 (SE 0.47) and 7.59 (SE 0.50). The mean patient-reported daily functioning in the CBT-based occupational therapy with sensor monitoring group was larger than that in the care as usual group (difference 1.17 [95\% CI (0.47-1.87) $P=$ $0.001]$. We found no significant differences in daily functioning between CBT-based occupational therapy and care as usual.

Conclusion: Among older patients recovering from hip fracture, a rehabilitation program of sensor monitoring-informed occupational therapy was more effec- 
tive in improving patient-reported daily functioning at six months than to care as usual. The findings have important implications for those active in the support of older patients after hip fracture. A successful implementation of this intervention, requires the use of goal setting and CBT-strategies to motivate patients to increase daily activities with the goal to recover. The objective information of the sensors provides professionals with more detailed information about daily activity patterns, so they can more effectively discuss goals and coach patients to recover.

Key words: Geriatric rehabilitation, sensor monitoring, cognitive behavioral treatment

*Corresponding author. E-mail: m.c.pol@hva.nl

\section{HABITAT: A New Generation of Ambient Assisted Living}

Alessandra Costanzo ${ }^{\mathrm{a}, *}$, Francesca Benassi ${ }^{\mathrm{b}}$, Elena Borelli $^{\mathrm{c}}$, Diego Masotti ${ }^{\mathrm{b}}$ and Giacomo Paolini ${ }^{\mathrm{b}}$

aDE "Guglielmo Marconi", University of Bologna, Via dell'Università 50, 47522 FC, Cesena, Italy

bDE "Guglielmo Marconi", University of Bologna, Viale del Risorgimento 2, 40136 BO, Bologna, Italy ${ }^{\mathrm{c}}$ CIRI SDV, University of Bologna, Via Tolara di Sopra 41/E, 40064 BO, Ozzano dell'Emilia (Bologna), Italy

Background: Over the last years, due to the increasing median age of the world population, solutions devoted to home living assistance are becoming a matter of urgency, enhancing interests throughout the scientific community. In particular, HABITAT (Home Assistance Based on the Internet of Things for the AuTonomy of Everybody) is a project addressed to families, caregivers, and to everyone who needs support in daily activities in order to guarantee increasing safety and to encourage the maintenance of a healthy lifestyle. The presented solution consists in a platform based on the typical technologies of Internet of Things (IoT), namely RFID (Radio Frequency IDentification), Wearable Electronics, Wireless Sensor Networks (WSN) and Artificial Intelligence, which allows to realize reconfigurable assistive environments.

Method: After an in-depth analysis of needs, carried out through interviews and focus groups involving the real users living in adult daycare centers, more than 450 requests were collected; based on these wishes, everyday life objects have been enhanced with brand new functionalities, with the aim of making them become "smart". As an example, a common wall lamp integrates an innovative indoor localization system, an armchair allows to detect the sitting posture and finally a belt incorporates a mobile health system able to perform an indoor and outdoor gait analysis. Concerning the indoor localization, a RFID reader (with maximum read range of $5.50 \mathrm{~m}$ and angular aperture of $\pm 45^{\circ}$ ) able to localize and track both people and objects equipped with RFID tags, has been developed. Thanks to the limited thickness of the reader, this technology is able to be inserted in any piece of furniture inside the house, i.e. hung on the wall inside a frame. Furthermore, a small RFID tag completes the overall system and can be worn by the users in a discrete way, like a brooch, a pendant, or with the antenna directly integrated in the textile materials. After preliminary laboratory investigations, 40-minute tests have been performed on 19 subjects, including caregivers, self-sufficient and non-self-sufficient seniors, from 55 to 83 years old, volunteers from a daycare facility on the outskirts of Bologna (Italy).

Key results: During the abovementioned tests, both the technology of the smart objects and their acceptability were validated and deeply investigated: with regards to the indoor localization, mean errors of about $18 \mathrm{~cm}$ were detected within a room of $32 \mathrm{~m}^{2}$. During the project, deep focus has been pointed towards the acceptability of the technology, together with the object design usability and their interaction with the final users.

Conclusion: In this work, a new version of AAL (Ambient Assisted Living) system addressed to elderly people living in their own houses or in daycare centers has been described; its future aims forecast improvements on the technology functionalities and on the user-friendly designs of the smart objects.

Keywords: Ambient Assisted Living, Elderly, Internet of Things, Assistive Technology.

*Corresponding author. E-mail: alessandra.costanzo@ unibo.it

LivingHub: An interdisciplinary approach to designing an innovative AT laboratory for ageing population by using simulation-based education

Niccolò Casiddu ${ }^{\mathrm{a}, *}$, Marco Chirico ${ }^{\mathrm{b}}$ and Claudia Porfirione $^{\mathrm{a}}$

${ }^{a}$ Department of Architecture and Design, University of Genova, Italy

${ }^{\mathrm{b}}$ University of Genova, Simav, Italy

Background: Simulation-based education methodology, born in the military field, has since earned 
widespread recognition in many fields as a powerful tool to strengthen knowledge, improve team communication and teach the skills of decision-making processes. Many Universities in the world have set up their own simulation laboratories, teaching and research centers, predominantly in engineering and medical areas. In particular, simulation based medical training enables students, physicians, nurses and other health professionals to integrate cognitive learning with practical experience, without posing risks to the patients. Today the use of simulation has surpassed military and medical applications and has allowed the development of transversal didactic teaching methods that could cover a wide range of new training needs.

Devised from the confluence of transdisciplinary skills (medicine, engineering, computer science, architecture, design) according to the AAL (Ambient Assisted Living) approach, this work presents the LivingHub project: a smart prototype apartment (about 70 square meters) to simulate domestic environments for ageing population integrated with systems of monitoring, control and assistance.

The LivingHub objective is to promote the spread of a new home healthcare culture that passes through simulation-based education, designing suitable spaces and devices and developing specific professional skills. Furthermore, the LivingHub wants to provide a reference model for new research and training opportunities in the field of interdisciplinary and interprofessional skills, across different scientific areas.

Method: Currently under construction, the LivingHub is being built as a movie set (folding triangular truss with audio-video-light system) consisting of four main rooms (bedroom, living room, bathroom, kitchen) and a control room.
The LivingHub is a home-laboratory (according to the model of the MIT Living Labs: http://livinglab.mit. $\mathrm{edu} /$ ) where different actors (coming from universities, companies, research centers, public institutions, citizens) have the chance to exchange ideas, following a multidisciplinary and user-centered approach able to promote innovation.

Starting from the detection of users' needs in carrying out daily activities, simulation training scenarios are built with technological aids by formal and informal caregivers, with particular attention to the concepts of accessibility and usability.

Key results: The LivingHub aims to create a hub that combines various functions, such as an educational laboratory for students and professionals, a showroom of technologies and services in AAL field, a research and development laboratory for assistive technologies and services.

Conclusion: The LivingHub not only aims to educate, through simulation, future highly qualified care professionals, but is also aimed at generating user-centered ecosystems, able to accelerate the large-scale adoption of assistive technologies and co-created services with the users themselves. The LivingHub encourages the integration of domotic technologies into the domestic environment with monitoring and remote assistance systems (telemedicine, tele-monitoring, robots for telepresence, remote data processing. ..).

Keywords: Simulation-based education, Interdisciplinary approach, AAL laboratory.

${ }^{*}$ Corresponding author. E-mail: casiddu@ arch.unige.it 